\title{
Terrestial laser scaning in forest invetory based on OptD optimization method
}

\author{
Joanna Janicka ${ }^{1, *}$, Wioleta Błaszczak-Bąk ${ }^{2}$, and Krystian Pawłowski ${ }^{1}$ \\ ${ }^{1}$ University of Warmia and Mazury in Olsztyn, Faculty of Geodesy, Geospatial and Civil Engineering, Oczapowskiego 2, 10-719 \\ Olsztyn, Poland
}

\begin{abstract}
Nowadays the time of acquiring spatial data is one of the most important parameters during measurements. Laser scanning technology makes it possible to record a huge number of measurement data in a relatively short time. Thus it is possible to acquire and update spatial data on large surface areas in a significantly shorter time compared to classical measurement methods. Terrestrial laser scanning can be used in forest inventory because measurements can acquire millimeter-level of detail. Thus measurement are performed quickly, automatically and provides periodical estimates of many important forest inventory attributes.
\end{abstract}

\section{Introduction}

Information about forest resources is collected for various purposes. A management and planning policy for these resources is conducted in forest areas. Depending on the purpose, size of the area and scale of the project, measurements are made in a different range. Measurements are made using different techniques and the required accuracy. The basic method of forest stand measurement is the classical method using tachometry. Unfortunately, this technique that is time-consuming and quite expensive. Of course, it provides high accuracy of measurement, but the task execution time is incomparable to the use of terrestrial laser scanning. Terrestrial laser scanning (TLS) automatically measures the surrounding three-dimensional (3D) space, using millions of $3 \mathrm{D}$ points [1].

The main advantage of using TLS in forest inventory is the possibility of fast, automatic and detailed forest documentation. The first work related to the estimation of tree attributes in forest inventory was recorded around 2000, e.g. [2-7]. The motivation to use TLS in forest inventory was to increase work efficiency on sample charts, i.e. to replace "manually" performed tree attribute measurements with those that were automatically taken from TLS data [8 -11].

Forest measurements using TLS can also be a useful tool for forest monitoring, i.e. periodic measurements on the basis of which the changes taking place over time can be assessed.

By referencing an object that has been subject to measurements, terrestrial laser scanning can also be used to update the map for design purposes. A fragment of the measurement results presented in the article presents the scanned area of the historic park belonging to the palace and park complex in Sztynort. The measurements were made in order to update the map for design purposes and as a basis for revitalization works planned for this object.
The scope of works included inventory of the forest and a number of analyzes conducted by greenery designers. The measurements were made using the classic method and using TLS. As it turned out, classical measurements took much more time at the field stage, while TLS measurements are carried out very quickly, while their later development is more complicated than in the case of classical data. A collection of measuring points from the so-called scanning "point cloud" is a huge collection of data that also contains a lot of unnecessary data from the environment. Therefore, the authors want to present the possibility of using OptD method of data optimization.

\section{OptD method}

The OptD is a optimization method described in [1214] and used to reducing the number of points in the processing of Airborne Laser Scanning point cloud. Of course there is a lot of algorithms described in the literature [15-16] that allows to filter and reduce the point clouds obtained from laser scanning, but the proposed algorithm, in comparison to other methods, allows to obtain the optimal solution. The algorithm of this method can reduce the datasets based on the specified optimization criterion. Such a criterion may be, for example, the percentage of points in the dataset after reduction or the standard deviation. The OptD method can be conducted in two options:

- Option 1: OptD method with one objective optimization (OptD-single).

- Option 2: OptD method with multi objective optimization (OptD-multi).

The most significant stage in OptD method is using the method of generalization. For this propose we used Opheim algorithm, which is often used in the

\footnotetext{
* Corresponding author: joannasuwm@gmail.com
} 
generalization of curves with complex shapes [17]. The points of a generalized object are examined by means of a tolerance figure built of a circle with a radius of $d_{\text {min }}$ and a surface with a length of $d_{\max }$. The initial value of the tolerances are defined by the user, the following values are determined in an iterative process.

If OptD-single method is chosen, then a set which strictly is fulfilling one condition is sought. There is also a possible to process using OptD-multi, then the several results will be obtained, among which the best one should be selected.

In this paper the OptD-single method was tested to optimize data from terrestrial laser scanning. For the OptD-single method the percentage of points to be left in the set after the reduction was chosen as the basic criterion. During the operation of the method, the following are selected: width of the measuring strip and tolerance used in Opheim generatlization method. The values of these parameters are calculated without user intervention and changed in the iterative process in such a way that the optimization criterion is met.

\section{Forest inventory with the OptD method}

Forest inventory performed by the classical method is a labor-intensive and time-consuming process. Before starting measurements, a geodetic network must be established or the existing one should be found. Unfortunately, GPS measurements cannot always be used to improve the measurements work because in the forested areas it is technically not possible. Then each tree is measured one by one. During measurements, it is important to mark the measured trees not to duplicate measurements or not to miss some.

Using terrestrial laser scanning may be helpful. TLS can be used to evaluate the tree attribute estimations, such as:

- Stem detection and stem density,

- DBH measurements,

- Tree height measurements

- Stem curve, volume and quality measurements

- Biomass estimation.

Thus, you can perform a series of analyses on the acquired data. The results depend on many factors, such as forest structures, the instrument used and processing methods. However, in any case of performed tests and analyses, it is important to pre-filtering the point cloud obtained as a result of the scanner measurement.

The proposed OptD method allows to significantly reduce the volume of data that is processed to obtain the final product, without losing their quality.

\section{Example}

Maps for design purposes are cartographic studies include the preparation of geodetic documentation necessary for the implementation of an investment project in Poland. The scope and mode of map execution are regulated by various legal acts which define in detail the final result of the entire process. Usually surveying works related to the map update task are associated with measurement in the field using classic measurement technologies such as tachometry or GNSS satellite measurements.

Updating the map for design purposes in the area of property located in Sztynort consisted in measuring trees: determining their location and determining selected features, such as height, diameter, etc. The area of the park is about 40 ha. The classic method ensures high accuracy of measurements, however, it is extremely time-consuming. The use of GPS technology was not possible because the crowns of trees obscured the visibility of the satellites. Therefore, an attempt was made to use also a technique of terrestrial laser scanning, in addition to the classical measurement technology.

As it was mentioned in the introduction a collection of measuring points from the scanning, "cloud of points", is a huge collection of data that also contains a lot of unnecessary data from the environment. Therefore, the authors want to present the possibility of using OptD methods of data set optimization.

The set of measurement points from scanning, "point cloud", is a huge data collection, which also contains a lot of unnecessary data from the environment. Therefore, the authors want to present the possibility of using OptD methods to optimize the data set.

The amount of data obtained as a result of scanning from forestry area (a lot of details, e.g. leaves, branches, shrubs) is very large, but incorrect reduction or filtration may result in the loss of valuable information. Therefore, it is important to optimize the data sets in such a way that with the minimum of data we retain maximum information.

The analyzes were carried out in three stages, successively for the forest area, single tree and trunk. The aims of performed tests were (a) to present how the reduction carried out on the entire (initial) dataset influences the set of points representing a single tree, and (b) does the tree after reduction retain its geometry, and the points representing the tree can be used for 3D modeling. This is particularly important for green area designers, biomass calculation, state and age of the forest.

In the first stage a forest fragment was used for the tests. This fragment was measured from three scanner stations, so the 3D image was composed of three scans. Selected forest fragment before processed the data with the use of OptD method included about seven hundred thousand points. Separation of individual tree trunks, and then modeling was quite complicated, because there were many observations that disturbed the picture. Thus, at the initial stage of the work, the collection was reduced using the OptD method. Optimization of the dataset at various levels was performed. The obtained results are presented in figure 1. 

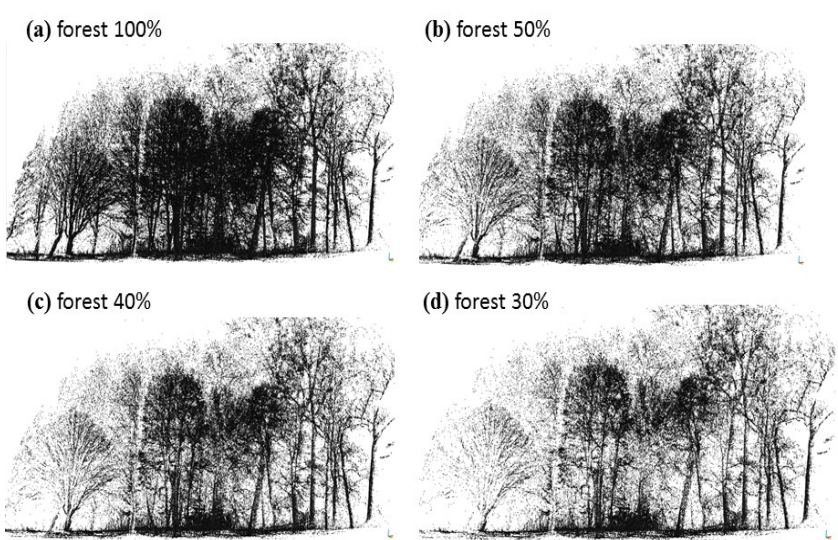

Fig. 1. Dataset without optimization with OptD method (forest 100\%) and after optimization wit OptD: a), b) and c).

The characteristics of obteined dataset after OptD method are presenetd in table 1 .

The following parameters were used to characterize the reduced datasets: Zmin, Zmax, Zmean, standard deviation (SD). The OptD method allowed keeping Zmin and Zmax values, the average value of the height in the set will change and the SD parameter means the range of the height of the measurement points in relation to the mean. SD will increase as the number of points in the point cloud decreases.

Table 1. Characteristics of obtained parameters for the forest.

\begin{tabular}{|l|l|l|l|l|l|}
\hline & \multicolumn{5}{|c|}{ Parameters } \\
\hline Dataset & $\begin{array}{l}\boldsymbol{Z}_{\min } \\
{[\mathbf{m}]}\end{array}$ & $\begin{array}{l}Z_{\text {max }} \\
{[\mathbf{m}]}\end{array}$ & $\begin{array}{l}\boldsymbol{Z}_{\text {mean }} \\
{[\mathbf{m}]}\end{array}$ & $\begin{array}{l}\text { Number } \\
\text { of points }\end{array}$ & $\begin{array}{l}\text { SD } \\
{[\mathbf{m}]}\end{array}$ \\
\hline $\begin{array}{l}\text { forest } \\
\mathbf{1 0 0 \%}\end{array}$ & -3.029 & 34.713 & 9.895 & 682467 & 7.683 \\
\hline $\begin{array}{l}\text { Forest } \\
\mathbf{5 0 \%}\end{array}$ & -3.029 & 34.713 & 10.410 & 341608 & 8.831 \\
\hline $\begin{array}{l}\text { Forest } \\
\mathbf{4 0 \%}\end{array}$ & -3.029 & 34.713 & 10.512 & 271489 & 9.219 \\
\hline $\begin{array}{l}\text { Forest } \\
\mathbf{3 0 \%}\end{array}$ & -3.029 & 34.713 & 10.642 & 202861 & 9.658 \\
\hline
\end{tabular}

The optimization was carried out in a few scenarios, leaving 50,40 and $30 \%$ of the starting set. The maximum reduction number of points was reduced to $30 \%$, so approximately 200,000 points. Unfortunately, the cloud of points, which still contains a huge amount of data, does not show clearly its features in a small drawing. The numerical data presented in the table 1 show some values, which confirm that even a large reduction of data does not worsen the quality of the resultant dataset.

The next stage of the study was to test whether the optimization using the OptD method allows to significant reduce the size of the data for a single tree but without losing the quality of the data. The number of points representing this single tree presented on the figure is more than twenty thousand points. And in the case of a single tree, you can reduce the size of the data set to $30 \%$ of the original size and still the full validity of the tested object is assured.

The figure 2 shows a single tree based on the original dataset and datasets after OptD method. The shape of the tree has been preserved, despite the use of various degrees of reduction.

The characteristics of obtained dataset after OptD method are presented in the table 2. The number of points has been reduced from 22,000 to less than 7,000, and the standard deviation has changed slightly.

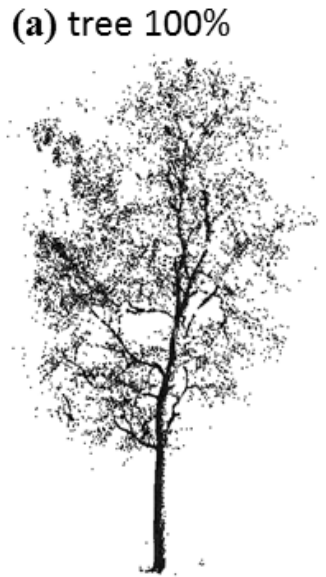

(c) tree $40 \%$

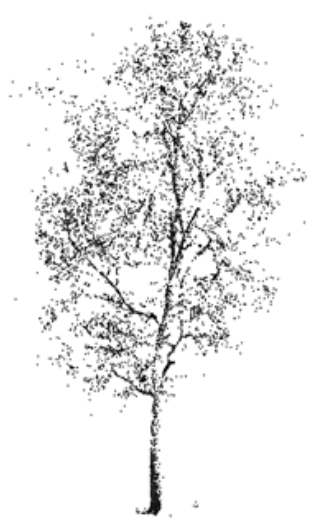

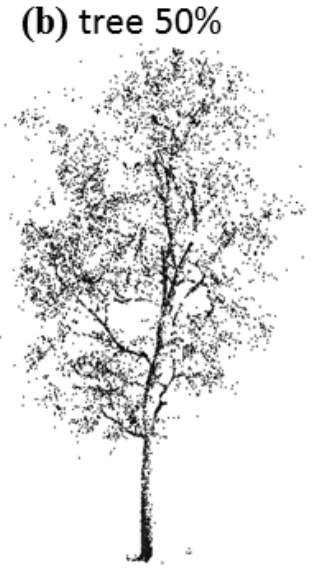

(d) tree $30 \%$

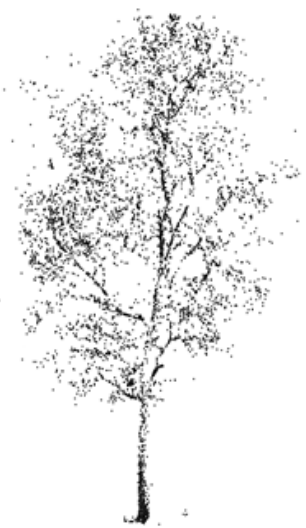

Fig. 2. Dataset without optimization with OptD method (tree $100 \%$ ) and after optimization wit OptD: a), b) and c).

Table 2. Characteristics of obtained parameters for a single tree.

\begin{tabular}{|c|c|c|c|c|c|}
\hline & \multicolumn{5}{|c|}{ Parameters } \\
\hline Dataset & $\begin{array}{c}\boldsymbol{Z}_{\text {min }} \\
{[\mathbf{m}]}\end{array}$ & $\begin{array}{c}Z_{\text {max }} \\
{[\mathrm{m}]}\end{array}$ & $\begin{array}{c}\boldsymbol{Z}_{\text {mean }} \\
{[\mathbf{m}]}\end{array}$ & $\begin{array}{c}\text { Number } \\
\text { of points }\end{array}$ & $\begin{array}{c}\text { SD } \\
{[\mathrm{m}]}\end{array}$ \\
\hline $\begin{array}{c}\text { Tree } \\
\mathbf{1 0 0 \%}\end{array}$ & -1.094 & 31.157 & 13.557 & 22008 & 7.458 \\
\hline $\begin{array}{c}\text { Tree } \\
\mathbf{5 0 \%}\end{array}$ & -1.094 & 31.157 & 15.457 & 10982 & 7.276 \\
\hline $\begin{array}{c}\text { Tree } \\
\mathbf{4 0 \%}\end{array}$ & -1.094 & 31.157 & 15.673 & 8886 & 7.431 \\
\hline $\begin{array}{c}\text { Tree } \\
\mathbf{3 0 \%}\end{array}$ & -1.094 & 31.157 & 15.919 & 6649 & 7.706 \\
\hline
\end{tabular}


The last stage of the study consisted in reducing the number of points representing the tree trunk. The authors wanted to show that after data reduction, even at the level of 70 from the original dataset, the diameter, perimeter or height of the trunk can still be accurately determined.

The results are presented in figure 3 .

$\begin{array}{llll}\text { (a) tree trunk } 100 \% & \text { (b) ree trunk } 50 \% & \text { (c) tree trunk } 40 \%\end{array}$

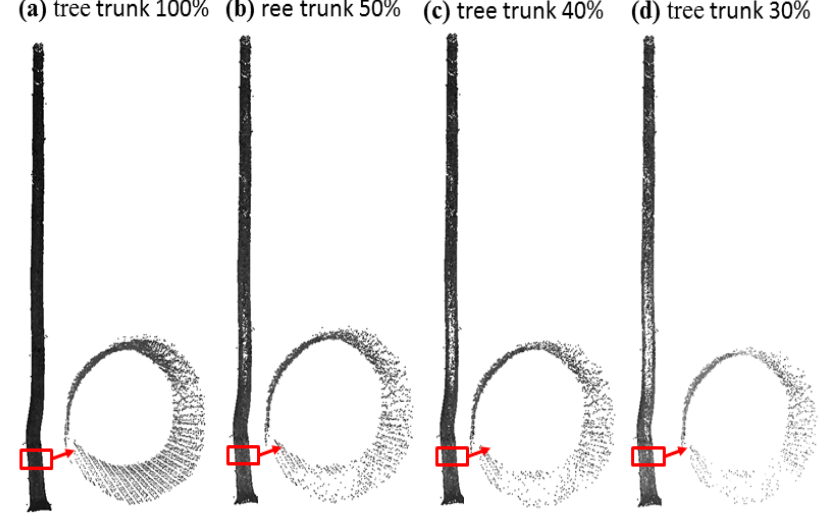

Fig. 3. Dataset without optimization with OptD method (forest $100 \%$ ) and after optimization wit OptD: a), b) and c).

As it was mentioned in the description of the method, it is important to preserve the three-dimensional features of the object. The results show that the crosssection of the tree trunk after the reduction of points to the level of $30 \%$ maintains the correct shape. The trunk perimeter calculated on the basis of the original data tree (trunk $100 \%$ ) at the height of $1.3 \mathrm{~m}$ is $1.36 \mathrm{~m}$. The same perimeter value was obtained for all reduced sets (trunk50\%, trunk $40 \%$, trunk30\%).

The characteristics of obtained dataset for tree trunk after OptD method are presented in the table 3.

Table 3. Characteristics of obtained parameters for tree trunk.

\begin{tabular}{|c|c|c|c|c|c|}
\hline & \multicolumn{5}{|c|}{ Parameters } \\
\hline Dataset & $\underset{[\mathrm{m}]}{Z_{\min }}$ & $\underset{[\mathrm{m}]}{Z_{\max }}$ & $\underset{[\mathrm{m}]}{Z_{\text {mean }}}$ & $\begin{array}{l}\text { Number } \\
\text { of points }\end{array}$ & $\begin{array}{l}\text { SD } \\
{[\mathrm{m}]}\end{array}$ \\
\hline $\begin{array}{l}\text { trunk } \\
100 \%\end{array}$ & -1.645 & 14.763 & 3.922 & 71158 & 4.057 \\
\hline $\begin{array}{c}\text { trunk } \\
50 \%\end{array}$ & -1.645 & 14.763 & 3.958 & 35409 & 4.573 \\
\hline $\begin{array}{c}\text { Trunk } \\
\mathbf{4 0 \%}\end{array}$ & -1.645 & 14.763 & 4.004 & 28503 & 4.774 \\
\hline $\begin{array}{c}\text { Trunk } \\
\mathbf{3 0 \%}\end{array}$ & -1.645 & 14.763 & 4.071 & 21507 & 5.024 \\
\hline
\end{tabular}

As in previous cases, extreme values of heights have been preserved, and the value of the SD parameter for the tree trunk has increased by $0.967 \mathrm{~m}$.

\section{Summary}

The aim of the study was to show that terrestrial laser scanning can be a valuable tool when performing forest inventory and study of single tree. Performing a scan of the area that is covered by the study is a quite simple task. Scanning takes a relatively short time, but at the also very important step is to develop the acquired data. In this paper OptD-single method was used in preliminary processing of TLS data. Performed analysis confirm the efficiency and effectiveness of the OptD method in the process of developing data from forest inventory. When significantly reducing the number of measured points, the remaining points represent the object with the appropriate accuracy. Therefore, any analysis can be performed without fear that the optimized dataset lost their quality. The details conclusions are: (a) OptD method can be used for entire dataset representing forest, but analysis can be performed for single object (tree, tree trunk), (b) the single tree after reduction preserved correct shape, (c) the same value of tree circuit was obtained for all reduced datasets.

\section{References}

1. X. Liang, V. Kankare, J. Hyyppä, Y. Wang, A. Kukko, H. Haggrén, X. Yu, H. Kaartine, A. Jaakkola, F. Guan, M. Holopainen, M. Vastaranta, Journal of Photogrametry and Remote Sensing, 115, Pages 63-77 (2016)

2. M. Erikson, V. Karin, Proceedings of the Scandlaser Scientific Workshop on Airborne Laser Scanning of Forest, pp. 244-250 (2003)

3. Lovell et al., Can. J. Remote Sens. 29 pp. 607-622 (2003) DOI:10.5589/m03-026

4. Hopkinson et al., Can. J. For. Res., 34 (2004), pp. 573-583, DOI:10.1139/x03-225

5. G.G. Parker, D.J. Harding, M.L. Berger, J. Appl. Ecol., 41 pp. 755-767 (2004) DOI:10.1111/j.00218901.2004.00925.x

6. M. Thies, N. Pfeifer, D. Winterhalder, B.G.H. Scand., J. For. Res., 19, pp. 571-581 (2004)

7. Henning and Radtke, Photogramm. Eng. Remote Sens., 72 p. 1349 (2006)

8. A. Bienert, S. Scheller, E. Keane, G. Mullooly, F. Int. Arch. Photogramm., Remote Sens. Spatial Inform. Sci., 36 (2006)

9. H.G. Maas, A. Bienert, S. Scheller, E. Keane, (2008). Int. J. Remote Sens., 29, pp. 1579-1593, Doi.10.1080/01431160701736406

10. M. Vastaranta, T. Melkas, M. Holopainen, H. Kaartinen, J. Hyyppä, H. Hyyppä, (2009). Photogramm. J. Finland, 21, pp. 51-61.

11. G.E. Murphy, M.A. Acuna, I. Dumbrel, (2010). Can. J. For. Res., 40, pp. 2223-2233 (2010) DOI:10.1139/X10-171 
12. W. Błaszczak-Bąk, Acta Geodynamica et Geomaterialia. 13, 4(184), pp. 379-386 (2016) DOI:10.13168/AGG.2016.0020.

13. W. Błaszczak-Bąk, A. Sobieraj-Żłobińska, M. Kowalik, Measurement Science of Technology, 8, 7, $075009 \quad$ (10pp). (2017) DOI:10.1088/13616501/aa7444

14. K. Bakuła , Challenges of Modern Technology, WUT PhD Students Board, 2, No. 4, pp. 31-36 (2011)

15. B. Bauer-Marschallinger, D. Sabel, W. Wagner, Computers \& Geosciences, 72, (2014) DOI:10.1016/j.cageo.2014.07.005

16. W. Błaszczak-Bąk, A. Sobieraj-Żłobińska, B. Wieczorek, E3S Web Conf. 26 00005, (2018), DOI: $10.1051 / \mathrm{e} 3$ sconf/20182600005

17. H. Opheim, Smoothing a digitized curve by data reduction methods. Eurographics '81, ed. by Encarnacao, J. L., pp. 127-135 (1981) 